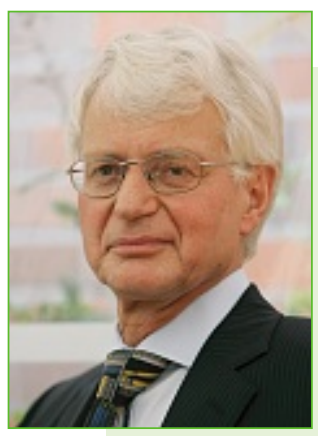

\title{
Arznei, die gefällt
}

"Placebo in der Medizin" - unter dieser Überschrift erschien am 19. Juli 2010 im Deutschen Ärzteblatt (DÄ) der Tätigkeitsbericht eines gleichnamigen Arbeitskreises der Bundesärztekammer (BÄK) (1). Vorausgegangen war ebenfalls im $D A ̈$ ein 5-seitiger Originalbeitrag mit dem Titel »Placebo: Missverständnisse und Vorurteile» (2). Das Leserecho, abgedruckt im DÄ vom 20. August 2010, war lebhaft. Im Grundsatz waren sich alle einig: Placebo-Wirkungen sind keine Randphänomene, sondern können erheblichen Anteil am Erfolg vieler Therapien haben. Die vertiefte Erforschung, Lehre und Nutzung innerhalb der Schulmedizin sei überfällig.

Weniger einvernehmlich waren die Meinungen zur praktischen Umsetzung dieser Vorsätze. In 4 von 8 Leserzuschriften wurde Anstoß an der Formulierung des BÄK-Arbeitskreises genommen, dass »PlaceboEffekte einen Teil, wenn nicht die Gesamtheit der Wirkungen der Komplementärmedizin“ (wozu auch die Phytotherapie gezählt wird) ausmachten. In Verbindung mit der ebenfalls im Bericht enthaltenen Aussage, dass gezielte Placebo-Gabe eine Täuschung der Patienten darstellen könnte, war sogar von einem indirekten Berufsverbot für naturheilkundlich orientierte Ärzte die Rede. Insbesondere dem Letzteren widersprachen die Autoren in ihrem Schlusswort vehement, wobei sie darauf verwiesen, dass der publizierte Bericht allein den Hinweis enthielt, dass der Einsatz von Placebo in der Schulmedizin unter Umständen eine rechtlich relevante Täuschung des Patienten darstellen könnte.

Letzteres trifft zweifellos auch zu. Offen bleibt dabei aber die Frage, welche Arzneimittel alternativ zu empfehlen sind für Anwendungsgebiete, bei denen Placebo-Wirkungen einen dominierenden Anteil am Therapieerfolg ausmachen (erwähnt wurden u.a. 80\% bei den Antidepressiva). Immerhin: Dem Autor dieses Editorials wurde ausdrücklich dafür gedankt,

\section{Editorial}

dass er in seiner Leserzuschrift die besondere Eignung von pflanzlichen Arzneimitteln für 10 typische Indikationen in die Diskussion gebracht hat. Darin liegt ein Ansatz, über den vor allem die Anbieter der mehr und mehr durch Misserfolge großer placebokontrollierter klinischer Studien in die Enge getriebenen Phytopharmaka nachdenken sollten.

„Die Pflanze» braucht bekanntlich zur vollen Entfaltung ihrer Wirksamkeit »das Wort». Der Zuspruch muss nicht immer vom Arzt ausgehen; Apotheker, Familienangehörige oder »die Medien« können ein Gleiches tun. Von den 3 weiteren Einflussgrößen "pharmakologisch bedingte Wirksamkeit", "Unbedenklichkeit» und »Kosten der Therapie» gewinnen allerdings im Rahmen der hier diskutierten Neuordnung die beiden letztgenannten Faktoren im gleichen Maße an Gewicht, wie die erstgenannte Größe zur Randfunktion wird. Daraus resultieren weitreichende Konsequenzen. Phytopharmaka, die als akzeptable Behandlungsalternativen im o.g. Sinne anerkannt werden wollen, müssen zukünftig ihre Unbedenklichkeit in jeder Hinsicht zweifelsfrei nach außen dokumentieren. Sie müssen aber auch den Preiswettbewerb mit guten Synthetika in gleicher Indikation bestehen können. Daraus erwachsen neue Maßstäbe an die Herstellung und Komposition der Wirkstoffe, die in vielen Fällen mit bisher gepflegten Grundsätzen und Maximen der Phytotherapie nicht mehr übereinstimmen dürften.

Volker Schulz

1 Bundesärztekammer. Stellungnahme des wissenschaftlichen Beirats der Bundesärztekammer "Placebo in der Medizin«. Deutsches Ärzteblatt 2010; 107: C1233-C1237

2 Breidert M, Hofbauer K. Placebo - Missverständnisse und Vorurteile. Deutsches Ärzteblatt 2009; 106: 751-755 